\title{
In Focus
Spotlight on the August 12 Issue
}

\author{
Robert A. Gross, MD, PhD, FAAN \\ Editor-in-Chief, Neurology ${ }^{\circledR}$
}

\section{Outcome after surgical or conservative management of cerebral cavernous malformations $\mathbb{a}$}

This observational, prospective, population-based cohort study is the largest comparison of microsurgical excision of predominantly supratentorial cerebral cavernous malformations (CCM) with a conservatively managed concurrent control group, describing functional outcome over 5 years. There was worse outcome after microsurgical excision of CCM in fully adjusted multivariable survival analyses of both primary and secondary outcomes. See p. 582

From editorialists Bernardini \& Stapf: “...this study provides compelling evidence that CCM excision may worsen short-term disability scores and increase the risk of symptomatic ICH and new neurologic deficits."

See p. 576

\section{Untreated brain arteriovenous malformation: Patient-level} meta-analysis of hemorrhage predictors :-

The authors conducted an individual patient data meta-analysis of 4 cohorts to identify risk factors for intracranial hemorrhage in the natural history course of brain arteriovenous malformations (AVMs). This analysis of 2,525 patients identified hemorrhagic presentation and increasing age as independent predictors of hemorrhage during follow-up. This study informs clinical practice for the management of brain AVMs where hemorrhage risk prediction is a primary determinant of all subsequent management decisions.

See p. 590

\section{Peripheral pulse measurement after ischemic stroke:}

\section{A feasibility study}

Simple pulse-taking was investigated as a screening tool for paroxysmal atrial fibrillation (AF) among 256 patients with ischemic stroke. As paroxysmal episodes are easily missed by a single ECG, the detection rate of AF depends on the modality and the duration of cardiac monitoring. The authors found that simple measurement of the peripheral pulse was feasible and accurately distinguished AF from normal heart rhythm when compared with continuous ECG as the gold standard. Along with socioeconomic expense, disability, and patient noncompliance risk, these results could inform the decision to refer individuals for extended cardiac rhythm monitoring.

See p. 598

Impaired corticopontocerebellar tracts underlie pseudobulbar affect in motor neuron disorders

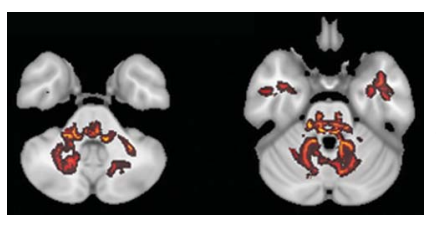

Pseudobulbar affect (PBA) adversely affects the quality of life, leading to social isolation and housebound status. The authors assessed how frequently PBA occurred in 50 patients with primary lateral sclerosis (PLS) over a

decade. They found that PBA is common among patients with PLS. Treatment can ameliorate symptoms of PBA and improve ratings of the quality of life in patients with PLS and amyotrophic lateral sclerosis.

See p. 620
Fulranumab for treatment of diabetic peripheral neuropathic pain: A randomized controlled trial

This phase II, randomized, double-blind, placebo-controlled, dose-ranging clinical trial evaluated the efficacy and safety of fulranumab, a fully human monoclonal antibody against nerve growth factor (NGF), in patients with diabetic peripheral neuropathic pain (DPNP). This study provided Class I evidence that in patients with DPNP, fulranumab $10 \mathrm{mg}$ reduced pain by 1.2 points on an 11-point scale compared with placebo. Long-term trials with more patients are needed to fully characterize the efficacy, safety, and tolerability of this new class of analgesic drug.

See p. 628

Lower neurocognitive function in U-2 pilots: Relationship to white matter hyperintensities

Subcortical white matter injury associated with occupational (military pilots) or avocational (high-altitude mountain climbers) exposure to hypobaric conditions can impair cognitive functioning. The authors collected neurocognitive data on United States Air Force (USAF) U2 pilots with a history of repeated exposure to hypobaria and compared them to control data collected from USAF pilots without repeated hypobaric exposure. Their results suggested that subcortical injury from any etiology-including mild traumatic brain injury-may impair performance on cognitive function testing in persons without apparent clinical impairment.

See p. 638

Multiple or mixed cerebral microbleeds and dementia in patients with vascular risk factors

In this prospective study, the authors investigated the association of cerebral microbleeds (CMBs) with dementia while controlling for other MRI findings. The dementia risk is higher for multiple or mixed CMBs, suggesting a dose-dependent or location-specific effect. CMBs are a surrogate marker for dementia risk depending on their load, which should prompt improved vascular care.

See p. 646

NB: Who or what killed Sally Wareham? Now you will know! Dr. Joynt's unfinished mystery "The case of the locked house" is now complete. The winning co-authors are Peter A. Kempster from Melbourne and Andrew J. Lees from London. See p. 661. 


\section{Neurology}

\section{Spotlight on the August 12 Issue}

Robert A. Gross

Neurology 2014;83;575

DOI 10.1212/WNL.0000000000000746

\section{This information is current as of August 11, 2014}

\section{Updated Information \&} Services

Permissions \& Licensing

Reprints including high resolution figures, can be found at: http://n.neurology.org/content/83/7/575.full

Information about reproducing this article in parts (figures,tables) or in its entirety can be found online at:

http://www.neurology.org/about/about_the_journal\#permissions

Information about ordering reprints can be found online:

http://n.neurology.org/subscribers/advertise

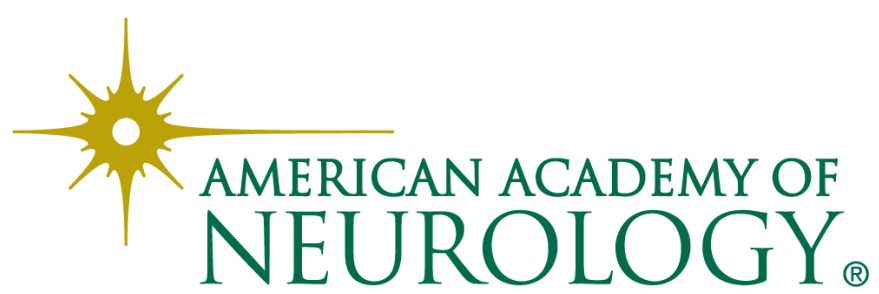

